\title{
Adding the Relation Meets to the Temporal Logic of Prefixes and Infixes Makes It EXPSPACE-Complete
}

\author{
Laura Bozzelli \\ University Federico II, \\ Naples, Italy \\ Department of Electric Engineering \\ and Information Technology \\ laura.bozzelli@unina.it
}

\author{
Angelo Montanari \\ University of Udine, Italy \\ Department of Computer Science, \\ Mathematics, and Physics \\ angelo.montanari@uniud.it \\ Pietro Sala \\ University of Verona, Italy \\ Department of Computer Science \\ pietro.sala@univr.it
}

\author{
Adriano Peron \\ University Federico II, \\ Naples, Italy \\ Department of Electric Engineering \\ and Information Technology \\ adriano.peron2@unina.it
}

\begin{abstract}
The choice of the right trade-off between expressiveness and complexity is the main issue in interval temporal logic. In their seminal paper [10], Halpern and Shoham showed that the satisfiability problem for HS (the temporal logic of Allen's relations) is highly undecidable over any reasonable class of linear orders. In order to recover decidability, one can restrict the set of temporal modalities and/or the class of models. In the following, we focus on the satisfiability problem for HS fragments under the homogeneity assumption, according to which any proposition letter holds over an interval if only if it holds at all its points. The problem for full $\mathrm{HS}_{\text {hom }}$ has been shown to be non-elementarily decidable [13], but its only known lower bound is EXPSPACE (in fact, EXPSPACE-hardness has been shown for the logic of prefixes and suffixes $B E_{h o m}$, which is a very small fragment of it [3]). The logic of prefixes and infixes $\mathrm{BD}_{\text {hom }}$ has been recently shown to be PSPACE-complete [5]. In this paper, we prove that the addition of the Allen relation Meets to $\mathrm{BD}_{\text {hom }}$ makes it EXPSPACE-complete.
\end{abstract}

\section{Introduction}

Interval temporal logics (ITLs for short) are versatile and expressive formalisms for specifying properties of sequences of states and their durations. When it comes to fundamental problems like satisfiability, their high expressive power is often paid at the price of undecidability. For instance, the most widely known ITLs, that is, HS logic, proposed by Halpern and Shoham's [10], and CDT logic, proposed by Venema [17], turn out to be highly undecidable w.r.t. the satisfiability problem. Despite these negative results, a number of decidable formalisms have been identified by weakening ITLs (see [6] for a complete classification of HS fragments). Here the term "weakening" is intended as a set of syntactic and/or semantics restrictions imposed on the formulas of the logic and/or the models on which such formulas are interpreted, respectively. Among the plethora of possible weakenings, in this paper we focus on (the combination of) the following two natural and well-studied restrictions:

- Restrict the set of interval relations. Many decidable fragments of ITLs are obtained by considering a restricted set of Allen's relations for comparing intervals. This approach naturally induces fragments of HS logic with modal operators corresponding to the selected subset of interval relations. As an example, the logic of temporal neighborhood, called PNL, features only two interval relations among the possible 13 ones, namely, $A$ (adjacent to the right) and its inverse $\bar{A}$. The

D. Bresolin and P. Ganty (Eds.): 12th International Symposium on Games, Automata, Logics, and Formal Verification (GandALF 2021) EPTCS 346, 2021, pp. 179-194, doi 10.4204/EPTCS.346.12
(C) L. Bozzelli, A. Montanari, A. Peron \& P. Sala This work is licensed under the Creative Commons Attribution License. 
corresponding interval modal logic has been shown to be decidable over basically every class of linear orders (e.g. see [7, 14]);

- Restrict the class of models. Based on a principle similar to the above one, some ITLs can be tamed by considering classes of models that satisfy certain specific assumptions. An example of this type of restriction can be found in a series of recent papers that studied model-checking problems for ITLs (e.g. see the seminal paper [13]), as well as ITL expressiveness compared to classical point-based temporal logics, like LTL, CTL, and CTL ${ }^{*}$ [2]. In this setting, models are represented as Kripke structures, and so inherently point-based rather than interval-based. The generated models can be equivalently obtained by making the so-called homogeneity assumption, that is, by assuming that every proposition letter holds over an interval if and only if it holds at all its points. It is important noticing that, under the homogeneity assumption, the full HS logic has a decidable satisfiability problem (as a matter of fact, the model-checking procedures introduced in the aforementioned works can be easily turned to satisfiability procedures, while often retaining the same complexity). Because of this, the focus in studying HS logics under the homogeneity assumption is shifted from decidability to complexity.

Let us focus now on the Chop logic $\mathrm{C}$ which is a proper fragment of CDT admitting as single modality called chop operator, that allows one to split the current interval in two parts and to require properties to hold separately on the two parts. In the general setting even the satisfiability problem for the logic $\mathrm{C}$ is undecidable, however if we impose the homogeneity constraint for satisfiability, thus obtaining the $\operatorname{logic} \mathrm{C}_{\text {hom }}$, then it can be easily shown that there is a LOGSPACE reduction of the emptiness problem for star-free generalized regular expressions to the satisfiability problem for $\mathrm{C}_{\text {hom }}$ and vice versa. However, a classic result in formal languages proved by Stockmeyer states that the emptiness problem for star-free generalized regular expressions is non-elementarily decidable (tower-complete) [15, 16]. This means that the satisfiability problem for $\mathrm{C}_{\text {hom }}$ is also non-elementarily decidable.

As we mentioned above, the satisfiability problem for the full logic HS when interpreted under the homogeneity assumption, called $\mathrm{HS}_{\text {hom }}$ from now on, is decidable [13], and the only known decision algorithm for such problem features a non-elementary complexity. To this day, the exact complexity of the satisfiability problem for $\mathrm{HS}_{\text {hom }}$ is still an open problem since a matching lower bound has not been fixed yet. From an expressivity standpoint, the aforementioned logic $C_{\text {hom }}$ can capture only three of the 12 modal operators featured by HS, namely, the operators $B$, for "begins", corresponding to the prefix relation on pairs of intervals, $D$, for "during", corresponding to the infix relation on pairs of intervals, and $E$, for "ends", corresponding to the suffix relation on pairs of intervals. It is easy to see that any fragment of $\mathrm{HS}_{\text {hom }}$ that contains both the operators $B$ and $E$ also contains $D$, since the infix relation may be expressed, for istance, as some prefix of some suffix or vice versa. Let us notice that the opposite is not true, e.g., the $\mathrm{BD}_{\text {hom }}$ fragment of $\mathrm{HS}_{\text {hom }}$ cannot express the $E$ operator. Informally speaking, this means that not all the properties about the prefixes of an interval cannot be expressed in terms prefixes and infixes. The satisfiability problem for the fragment $B E_{\text {hom }}$ has been proved to be EXPSPACE-hard in [3] while the upper bound ranges from EXPSPACE to non-elementary. We conjecture that the complexity of the satisfiability problem of $B E_{\text {hom }}$ coincides with the complexity of the problem of the full logic $\mathrm{HS}_{\text {hom }}$.

A couple of arguments that make $\mathrm{BE}_{h o m}$ such a peculiar beast are the following: (i) according to the results proved/summarized in [3] w.r.t. the satisfiability problem the only known fragments of $H S_{\text {hom }}$ for which it has been possible to provide an EXPSPACE lower bound for the complexity must contain both $B$ and $E$ operators; (ii) the satisfiability problem for the logic $\mathrm{DE}_{\text {hom }}$ (and it symmetric $\mathrm{BD}_{\text {hom }}$ ), which is a maximal proper fragment of $\mathrm{BE}_{\text {hom }}$ has been recently proved to be PSPACE-complete (see [1, 4, 5]). In this paper, we provide the first known fragment of $\mathrm{HS}_{\text {hom }}$ not including both the prefix and 
suffix modalities (B and E) in the EXPSPACE-completeness class. Such a fragment is the logic $\mathrm{ABD}_{\text {hom }}$ which is the extension of $\mathrm{BD}_{\text {hom }}$ with the meet modality A relating pairs of intervals where one interval begins exactly where the other ends).

In this paper we provide two main novel results: (i) we prove that the satisfiability problem for $\mathrm{ABD}_{\text {hom }}$ is EXPSPACE-hard on finite models by a reduction to the exponential corridor tiling problem; (ii) we provide a small model theorem for finite models of $\mathrm{ABD}_{\text {hom }}$ formulas that is doubly exponential in the size of the input formula. Then, by means of such small model theorem, we prove that there exists a decision procedure for the satisfiability problem of $\mathrm{ABD}_{\text {hom }}$ formulas that works using only exponential space w.r.t. the size of the input formula.

The paper is structured as follows. In Section 2 , we introduce syntax and semantics of $A B D_{\text {hom }}$ under the homogeneity assumption. In Section 3 we prove that the satisfiability problem for $\mathrm{ABD}_{\text {hom }}$ interpreted over finite models is EXPSPACE-hard. In Section 4 we introduce the notion of homogeneous compass structure, that provides a particularly useful representation for models of $A B D_{\text {hom }}$ formulas. In Section 5 , we give an EXPSPACE decision procedure for checking the satisfiability of $A B D_{\text {hom }}$ formulas. Finally, in Section 6, we provide an assessment of the work done and outline future research directions.

\section{The logic $A B D_{\text {hom }}$}

In this section, we introduce the logic $\mathrm{ABD}_{\text {hom }}$ and we define the satisfiability relation under the homogeneity assumption.

$\mathrm{ABD}_{\text {hom }}$ formulas are built up from a countable set Prop of proposition letters according to the following grammar: $\varphi::=p|\neg \psi| \psi \vee \psi|\langle A\rangle \psi|\langle B\rangle \psi \mid\langle D\rangle \psi$, where $p \in$ Prop and $\langle A\rangle,\langle B\rangle$, and $\langle D\rangle$ are the modalities for Allen's relations Adjacent (meets), Begins, and During, respectively.

Let $N \in \mathbb{N}$ be a natural number and let $\mathbb{I}_{N}=\{[x, y]: 0 \leq x \leq y \leq N\}$ be the set of all intervals over the prefix $0 \ldots N$ of $\mathbb{N}$. A (finite) model for BD formulas is a pair $\mathbf{M}=(N, \mathcal{V})$, where $\mathcal{V}: \mathbb{I}_{N} \rightarrow 2^{\text {Prop }}$ is a valuation that maps intervals in $\mathbb{I}_{N}$ to sets of proposition letters. Let $\mathbf{M}$ be a model and $[x, y]$ an interval. The semantics of a $\mathrm{ABD}_{\text {hom }}$ formula is defined as follows:

- $\mathbf{M},[x, y] \vDash p$ iff $p \in \mathcal{V}([x, y])$;

- $\mathbf{M},[x, y] \vDash \neg \psi$ iff $\mathbf{M},[x, y] \not \neq \psi$;

- $\mathbf{M},[x, y] \vDash \psi_{1} \vee \psi_{2}$ iff $\mathbf{M},[x, y] \vDash \psi_{1}$ or $\mathbf{M},[x, y] \vDash \psi_{2}$;

- $\mathbf{M},[x, y] \vDash\langle A\rangle \psi$ iff there is $y^{\prime}$, with $y^{\prime} \geq y$, such that $\mathbf{M},\left[y, y^{\prime}\right] \vDash \psi$;

- $\mathbf{M},[x, y] \vDash\langle B\rangle \psi$ iff there is $y^{\prime}$, with $x \leq y^{\prime}<y$, such that $\mathbf{M},\left[x, y^{\prime}\right] \vDash \psi$;

- $\mathbf{M},[x, y] \vDash\langle D\rangle \psi$ iff there are $x^{\prime}$ and $y^{\prime}$, with $x<x^{\prime} \leq y^{\prime}<y$, such that $\mathbf{M},\left[x^{\prime}, y^{\prime}\right] \vDash \psi$.

The logical constants $T$ (true) and $\perp$ (false), the Boolean operators $\wedge, \rightarrow$, and $\leftrightarrow$, and the (universal) dual modalities $[A],[B]$, and $[D]$ can be derived in the standard way. Moreover, it turns out to be useful to define: a constant $\pi=[B] \perp$ that holds only on intervals of the type $[x, x]$ (i.e., points); a global operator $[G] \psi=\psi \wedge[A] \psi \wedge[B] \psi \wedge[B][A] \psi$ in order to impose constraints on all the intervals in the model. We say that a $\mathrm{ABD}_{\text {hom }}$ formula $\varphi$ is (finitely) satisfiable if and only if there exist a model $\mathbf{M}=(N, \mathcal{V})$ and an interval $[x, y]$ such that $\mathbf{M},[0, N] \vDash \varphi$. We say that a model $\mathbf{M}=\left(\mathbb{I}_{N}, \mathcal{V}\right)$ is homogeneous if $\mathcal{V}$ satisfies the following property:

$$
\forall p \in \text { Prop } \forall[x, y] \in \mathbb{I}_{N}(p \in \mathcal{V}([x, y]) \Leftrightarrow \forall z \in[x, y] p \in \mathcal{V}([z, z])) .
$$


In Fig. 1, we show a homogeneous model (a) and a nonhomogeneous one (b). In homogeneous models, for any proposition letter, the labelling of point-intervals determines that of arbitrary intervals. This is not the case with arbitrary models (see, e.g., [4,6]). As a consequence, in homogeneous models, the labelling of the intersection of two intervals contains the labellings of the two intervals (this is the case with intervals $[1,6]$ and $[4,7]$ in Fig. 1 (a), whose intersection is the interval $[4,6])$. Once again, this is not the case with arbitrary models (see the very same intervals in Fig.1(b)).

Satisfiability can be recast in the case of homogeneous models. We say that a $\mathrm{ABD}_{\text {hom }}$ formula $\varphi$ is satisfiable under homogeneity if there is a homogeneous model $\mathbf{M}$ such that $\mathbf{M},[0, N] \vDash \varphi$.

Satisfiability under homogeneity is clearly more restricted than plain satisfiability. We know from [11, 12] that dropping the homogeneity assumption makes $D$ undecidable. This is not the case with the fragment $B$ that, being extremely weak in terms of expressive power, remains decidable [9]. The rest of

(a)
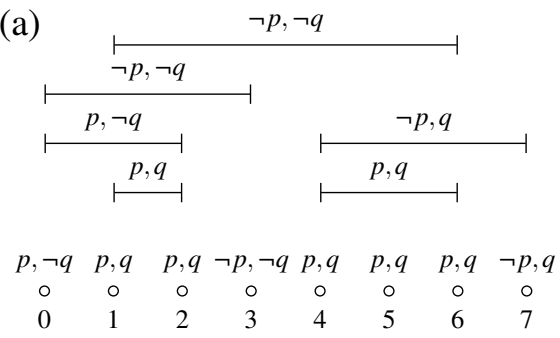

(b)

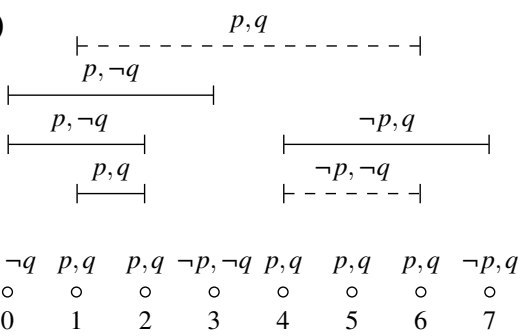

Figure 1: A homogeneous model (a above) vs. a general one (b-below). this paper is devoted to prove the following theorem.

Theorem 1. Given a $\mathrm{ABD}_{\text {hom }}$ formula $\varphi$ the problem of deciding whether or not there exists an homogeneous model $\mathbf{M}=(N, \mathcal{V})$ s.t. $\mathbf{M},[0, N] \vDash \varphi$ is an EXPSPACE-complete problem.

The proof of Theorem 1 is distributed over the next three sections. First, in Section 3 , we prove that such problem is EXPSPACE-hard, then, in Section 4 we provide a representation of homogeneous models, called compass structure, exploited in Section 5 to design an EXPSPACE decision procedure for the satisfiability problem of $\mathrm{ABD}_{\text {hom }}$ formulas.

\section{EXPSPACE-hardness for the logic $A B D_{h o m}$ over finite linear orders}

In this section we prove that the satisfiability problem for $\mathrm{ABD}_{\text {hom }}$ interpreted over finite linear orders is EXPSPACE-hard. The result is obtained by a reduction from the exponential-corridor tiling problem, which is known to be EXPSPACE-complete [8]. Such a problem can be stated as follows.

Problem 1. Given a tuple $\mathcal{T}=(T, \Rightarrow, \Uparrow, C)$ where $T, C \in \mathbb{N}$ (C is expressed in binary), and $\Rightarrow, \Uparrow \subseteq$ $\{0, \ldots, T\} \times\{0, \ldots, T\}$, the exponential-corridor tiling problem consists of determining whether or not there exists a function tile $: \mathbb{N} \times\{0, \ldots, C\} \rightarrow\{0, \ldots, T\}$ such that:

1. for every $x \in \mathbb{N}$ we have tile $(x, 0)=0$ and tile $(x, C)=T$;

2. for every $x \in \mathbb{N}$ and every $0 \leq y \leq C$ we have $($ tile $(x, y)$, tile $(x+1, y)) \in \Rightarrow$;

3. for every $x \in \mathbb{N}$ and every $0 \leq y<C$ we have (tile $(x, y)$, tile $(x, y+1)) \in \Uparrow$.

The following classical result will be exploited to prove the main goal of this section.

Theorem 2. [8] The exponential-corridor tiling problem is EXPSPACE-hard.

For defining a reduction from Problem 1 to the finite satisfiability of $A B D_{h o m}$ we have to deal with the problem that the formulas of $\mathrm{ABD}_{\text {hom }}$ are interpreted over finite domains whereas the tile functions ranges over an infinite domain. Roughly speaking, we shall solve Problem 1 by means of an infinite "unfolding" of a finite portion of the tiling space that can be encoded by a (finite) model for a suitable $\mathrm{ABD}_{\text {hom }}$ formula. The following result is crucial to that purpose. 
Lemma 1. Given an instance $\mathcal{T}=(T, \Rightarrow, \Uparrow, C)$ of Problem $\square$ we have that $\mathcal{T}$ is a positive instance if and only if there exists a function tile $: \mathbb{N} \times\{0, \ldots, C\} \rightarrow\{0, \ldots, T\}$ that fulfills conditions 12 and 3 of Problem 1 together with the following one:

4. there exist prefix $\in \mathbb{N}$ and period $\in \mathbb{N}^{+}$s.t. for every $x \geq$ prefix and every $0 \leq y \leq C$ we have tile $(x, y)=$ tile $(x+$ period,$y)$.

The proof of Lemma1 is straightforward and omitted. Lemma1 allows us to bound the search space for the existence of the function tile to a finitely representable function $\overline{\text { tile }}:\{0, \ldots$, prefix, $\ldots$, prefix + period $\} \rightarrow\{0, \ldots, T\}$ for some prefix $\geq 0$ and period $>0$. Function $\overline{\text { tile }}$ witnesses that $\mathcal{T}$ is a positive instance of Problem 1 if it satisfies conditions 1, 2, and 3 restricted to $(x, y) \in \mathbb{N} \times\{0, \ldots, C\}$ with $x<$ prefix + period plus the condition that $\overline{t i l e}($ prefix,$y)=\overline{\text { tile }}($ prefix + period,$y)$ for every $y \in\{0, \ldots, C\}$.

Given an instance $\mathcal{T}=(T, \Rightarrow, \Uparrow, C)$ of Problem 1 we provide a $\mathrm{ABD}_{\text {hom }}$ formula $\varphi_{\mathcal{T}}$ that is satisfiable over finite models if and only if there exists a function $\overline{\text { tile }}$ that satisfies the aforementioned properties an thus, by Lemma1, if and only if $\mathcal{T}$ is a positive instance of Problem 1. In the proposed encoding we force each point of the model to represent exactly one tile. This is done by exploiting $T+1$ propositional variables $t_{0}, \ldots, t_{T}$, called tile variables, constrained by the following formulas:

$$
\begin{aligned}
& \psi_{\exists}=[G]\left(\pi \rightarrow \bigvee_{i=0}^{T} t_{i}\right), \text { given a point in the model at least one tile variable holds over it; } \\
& \psi_{!}=[G]\left(\bigwedge_{i=0}^{T}\left(t_{i} \wedge \pi \rightarrow\left(\bigwedge_{j=0, j \neq i}^{T} \neg t_{j}\right)\right)\right), \begin{array}{l}
\text { given a point in the model at most one tile variable } \\
\text { holds over it (i.e., mutual exclusion). }
\end{array}
\end{aligned}
$$

Let us assume w.l.o.g. that $C=2^{c}-1$ for some $c \in \mathbb{N}$. Then, we associate to each model point a number in $\{0, \ldots, C\}$ by a binary encoding via $c$-propositional variables $b_{1}, \ldots, b_{c}$, where $b_{1}$ is the most significative bit. Formally, given a model $\mathbf{M}=(N, \mathcal{V})$ and a point we define a function with

$$
\text { bit }_{\mathcal{V}}:\{0, \ldots, N\} \times\left\{b_{1}, \ldots, b_{c}\right\} \rightarrow\{0,1\} \text { where } \operatorname{bit}_{\mathcal{V}}\left(n, b_{i}\right)=\left\{\begin{array}{ll}
1 & \text { if } b_{i} \in \mathcal{V}([n, n]) \\
0 & \text { otherwise }
\end{array} .\right.
$$

For the sake of brevity, we denote with $\bar{y}_{n}$ the natural number whose $c$-bit length binary encoding is bit $_{\mathcal{V}}\left(n, b_{1}\right) \ldots$ bit $_{\mathcal{V}}\left(n, b_{c}\right)$. We encode the domain of a general function $\overline{\text { tile }}:\{0, \ldots$, prefix, $\ldots$, prefix + period $\} \rightarrow\{0, \ldots, T\}$ into a finite model $\mathbf{M}=(N, \mathcal{V})$ by enumerating all the points of the grid $\{0, \ldots$, prefix + suffix $\} \times\{0, \ldots, C\}$ along the timepoints $\{0, \ldots, N\}$ of the model in a lexicographical order. The formula $\psi_{\text {tile }}=\psi_{\exists} \wedge \psi_{!} \wedge \psi_{\text {boundaries }} \wedge \psi_{\uparrow}$ is used to force such constraint where $\psi_{\text {boundaries }}$ and $\psi_{\uparrow}$ are formulas defined as follows:

$$
\begin{array}{ll}
\psi_{\text {boundaries }}=\langle B\rangle\left(\pi \wedge \bigwedge_{i=1}^{c} \neg b_{i}\right) \wedge[A]\left(\bigwedge_{i=1}^{c} b_{i}\right), & \begin{array}{l}
\text { every model } \mathbf{M}=(N, \mathcal{V}) \text { for } \psi_{\text {boundaries }} \text { satisfies } \bar{y}_{0}=0 \\
\text { and } \bar{y}_{N}=C ;
\end{array} \\
\psi_{\uparrow}=[G]\left([B] \pi \rightarrow\left(\bigwedge_{i=1}^{c}\langle B\rangle b_{i} \wedge\left([A] \perp \vee \bigwedge_{i=1}^{c}\langle A\rangle\left(\pi \rightarrow \neg b_{i}\right)\right)\right) \vee \psi_{+}^{1}\right), & \begin{array}{l}
\text { for every } n \in\{0, \ldots, N\} \text { if } \bar{y}_{n}= \\
\text { if } \bar{y}_{n}<N \text { then } \bar{y}_{n+1}=\bar{y}_{n+1}+1
\end{array}
\end{array}
$$
${ }_{i} \quad\left(\langle B\rangle b_{i} \rightarrow\langle A\rangle\left(\pi \wedge \neg b_{i}\right) \wedge \psi_{+}^{i+1}\right) \wedge \quad$ formula $\psi_{+}^{i}$ encodes the bit-wise increment for every bit $\psi_{+}^{i}=\begin{aligned} & \left(\langle B\rangle b_{i} \rightarrow\langle A\rangle\left(\pi \wedge \neg b_{i}\right) \wedge \psi_{+}^{i+1}\right) \wedge \\ & \left(\langle B\rangle \neg b_{i} \rightarrow\langle A\rangle b_{i} \wedge \psi_{=}^{i+1}\right)\end{aligned} \quad, \quad b_{i}$ with $i \in\{1, \ldots, c-1\} ; \psi_{+}^{1}$ is triggered by $\psi_{\uparrow}$ on every interval $[n, n+1]$ with $\bar{y}_{n}<C$;

$\psi_{+}^{c}=\neg\langle B\rangle b_{i} \wedge\langle A\rangle b_{i}, \quad$ formula $\psi_{+}^{c}$ encodes the bit-wise increment for the bit $b_{c}$; it is triggered by $\psi_{+}^{c-1}$ let us notice that it does not propagate and it handles overflows by creating a contradiction;

$$
\psi_{=}^{i}=\neg \pi \wedge \bigwedge_{j=i}^{j=c}\left(\langle B\rangle\left(\pi \wedge b_{i}\right) \leftrightarrow\langle A\rangle\left(\pi \wedge b_{i}\right)\right), \begin{aligned}
& \text { formula } \psi_{=}^{i} \text { holds over an interval }\left[n, n^{\prime}\right] \text { if and only if } \\
& n<n^{\prime} \text { and bit } t_{\mathcal{V}}\left(n, b_{j}\right)=\operatorname{bit}\left(n^{\prime}, b_{j}\right) \text { for every } i \leq j \leq c ;
\end{aligned}
$$


Note that if $\psi_{=}^{1}$ holds over $\left[n, n^{\prime}\right]$ then $\bar{y}_{n}=\bar{y}_{n^{\prime}}$. Formula $\psi_{=}^{i}$ is used for guaranteeing the correct bitwise increment in formulas $\psi_{+}^{i}$, moreover it will be used in the following for correctly identifying tiles which are in the $\Rightarrow$ relation.

It is worth noticing that any model $\mathbf{M}=(N, \mathcal{V})$ that satisfies $\psi_{\text {tile }}=\psi_{\exists} \wedge \psi_{!} \wedge \psi_{\text {boundaries }} \wedge \psi_{\uparrow}$ fulfills some properties. First of all, the interplay between $\psi_{\text {boundaries }}$ and $\psi_{\uparrow}$ guarantees that $N$ is a multiple of $(C+1)$ and thus, for suitably chosen prefix and suffix, we can associate each point $(x, y) \in\{0, \ldots$, prefix + suffix $\} \times\{0, \ldots, C\}$ to a point $n \in\{0, \ldots, N\}$ by means of a bijection map : $\{0, \ldots$, prefix + suffix $\} \times\{0, \ldots, C\} \rightarrow\{0, \ldots, N\}$ defined as $\operatorname{map}(x, y)=x \cdot(C+1)+y$ (i.e., map $^{-1}(n)=\left(\left\lfloor\frac{n}{C+1}\right\rfloor, n \% C\right)$ where $\%$ is the integer remainder operation). Moreover, let us observe that for every element $(x, y)$ in the grid, we have that $x$ is just implicitly encoded in the model by $\operatorname{map}(x, y)$ (i.e., $x=\left\lfloor\frac{\operatorname{map}(x, y)}{C+1}\right\rfloor$ ), while $y$ is both implicitly encoded (i.e., $x=\lfloor\operatorname{map}(x, y) \% C$ ) and explicitly encoded by the the values of variables $b_{1} \ldots b_{c}$ since it is easy to prove that $\psi_{\text {boundaries }} \wedge \psi_{\uparrow}$ forces $y=\bar{y}_{\text {map }(x, y)}$. Finally, the conjuncts $\psi_{\exists} \wedge \psi_{\text {! }}$ ensure that each point in $n \in\{0, \ldots, N\}\}$, and thus, by means of map, any point in the grid, is associated with exactly one tile, that is the unique tile variable that belongs to $\mathcal{V}([n, n])$.

For the aforementioned properties, if we consider the function $f$ that maps a function $\overline{\text { tile }}:\{0, \ldots, M\}$ $\times\{0, \ldots, C\} \rightarrow\{0, \ldots, T\}$ in the model $\mathbf{M}=(M \cdot(C+1), \mathcal{V})$ where for every $(x, y) \in\{0, \ldots, M\} \times$ $\{0, \ldots, C\}$ we have $t_{i} \in \mathcal{V}([\operatorname{map}(x, y), \operatorname{map}(x, y)])$ if and only if $\overline{t i l e}(x, y)=i$ and $\bar{y}_{\operatorname{map}(x, y)}=y$, it is easy to prove that $f$ is a bijection between the set of all such $\overline{t i l e}$ function, for every $M \in \mathbb{N}^{+}$, and the set of all finite models for $\psi_{\text {tile }}$. In summary, the detailed description above shows that any model for $\psi_{\text {tile }}$ is basically a way to represent a generic function $\overline{t i l e}:\{0, \ldots, M\} \times\{0, \ldots, C\} \rightarrow\{0, \ldots, T\}$ and that, viceversa, each of such functions is represented by exactly one model of $\psi_{\text {tile }}$. The next step is the encoding of the constraints of Lemma 1 in $\mathrm{AB}_{\text {hom }}$ which allow to check whether there exists a function $\overline{\text { tile }}$ that witnesses that $\mathcal{T}$ is a positive instance. Such conditions, restricted to the finite case, are imposed by the following formulas:

$\psi_{0, C}=[G]\left(\left(\left(\pi \wedge \bigwedge_{i=1}^{C} \neg b_{i}\right) \rightarrow t_{0}\right) \wedge\left(\left(\pi \wedge \bigwedge_{i=1}^{C} b_{i}\right) \rightarrow t_{T}\right)\right) \begin{aligned} & \text { formula } \psi_{0, C} \text { forces condition } 1 \text { of Prob- } \\ & \text { is } 0 \text { and the top tile of each column is } T ;\end{aligned}$

$\psi_{\Rightarrow}=[G]\left(\pi \wedge\langle A\rangle \neg \pi \rightarrow\langle A\rangle\left(\psi_{=}^{\min } \wedge\left(\bigvee_{(i, j) \in \Rightarrow}\left(\langle B\rangle t_{i} \wedge\langle A\rangle t_{j}\right)\right)\right)\right), \begin{aligned} & \text { formula } \psi_{\Rightarrow} \text { forces condition[ } 2 \text { of } \\ & \text { Problem 1, that is, each pair of }\end{aligned}$ grid points of type $(x, y),(x+1, y)$ must be labelled with two tiles that are in the $\Rightarrow$ relation. This is done by taking for each point $n<N$ the minimal interval $\left[n, n^{\prime}\right]$ with $n<n^{\prime}$ and $\bar{y}_{n}=\bar{y}_{n^{\prime}}$; then, the $\Rightarrow$ relation is forced between the pair of tile variables that hold over $[n, n]$ and $\left[n^{\prime}, n^{\prime}\right]$, respectively;

$\psi_{=}^{\min }=\psi_{=}^{1} \wedge[B] \neg \psi_{=}^{1}$, formula $\psi_{=}^{\min }$ holds over an interval $\left[n, n^{\prime}\right]$ if and only if $n<n^{\prime}, \bar{y}_{n}=\bar{y}_{n^{\prime}}$, and $\psi=\wedge[B] \neg \psi_{=}^{\prime}$, does not exist $n<n^{\prime \prime}<n^{\prime}$ such that $\bar{y}_{n}=\bar{y}_{n^{\prime \prime}}$. Let us notice that, for the constraints imposed by $\psi_{\text {tile }}$ we have that $n^{\prime}-n=C+1$ and thus, according to the definition of map, we have map $^{-1}\left(n^{\prime}\right)=\left(\left\lfloor\frac{n}{C+1}\right\rfloor+1, n \% C\right)$; then, $\psi_{=}^{\min }$ holds on all and only those intervals whose endpoints represent horizontally adjacent points of the original grid;

$\psi_{\Uparrow}=[G]\left([B] \pi \wedge \bigvee_{i=1}^{c} \neg b_{i} \rightarrow \bigvee_{(i, j) \in \Uparrow}\left(\langle B\rangle t_{i} \wedge\langle A\rangle t_{j}\right)\right)$,

formula $\psi_{\Uparrow}$ forces condition 3 of Problem 1 , that is, each pair of grid points of type $(x, y),(x, y+1)$ must be labelled with two tiles that are in the $\Uparrow$ relation. The constraint can be easily imposed 
since the encoding ensures that vertical consecutive points in the grid corresponds to consecutive points in the model. The constraint is triggered on all the intervals of the type $[n, n+1]$, with the exception of the of the ones with $\bar{y}_{n}=C$. The constraint imposes that unique (thanks to $\psi_{\exists} \wedge \psi_{!}$) pair of tile variables $\left(t_{i}, t_{j}\right)$ with $\left(t_{i}\right) \in \mathcal{V}([n, n])$ and $\left(t_{j}\right) \in \mathcal{V}\left(\left[n^{\prime}, n^{\prime}\right]\right)$ must satisfy $(i, j) \in \Uparrow$.

$\psi_{\text {prefix }}=\begin{gathered}\langle B\rangle\langle A\rangle\left(p \wedge \bigwedge_{i=1}^{C}\left(\langle B\rangle\left(\pi \wedge \neg b_{i}\right) \wedge\langle A\rangle b_{i}\right)\right) \wedge \\ {[G]\left(p \wedge \pi \rightarrow\langle A\rangle\left(\psi_{=}^{1} \wedge[A] \neg \psi_{=}^{1} \wedge \bigwedge_{i=0}^{T}\left(\langle B\rangle t_{i} \leftrightarrow[G] t_{i}\right)\right)\right)}\end{gathered}$

formula $\psi_{\text {prefix }}$ forces condition 4 of Lemma 1 , which imposes that there are two distinct columns in the grid which are tiled identically and one of such columns is the last one.

This is done by means of a propositional letter $p$. The first conjunct of formula $\psi_{\text {prefix }}$ imposes that there exists an interval $\left[n, n^{\prime}\right]$ in the model for which $p \in \mathcal{V}\left(\left[n, n^{\prime}\right]\right), \bar{y}_{n}=0$, and $\bar{y}_{n^{\prime}}=C$ (i.e., $p$ "covers" at least one column). Moreover, for the homogeneity assumption, we have that $p \in \mathcal{V}\left(\left[n^{\prime \prime}, n^{\prime \prime}\right]\right)$ for every $n \leq n^{\prime \prime} \leq n^{\prime}$. The second conjunct imposes that for each $p$ labelled points $n$ there must exist a point $n^{\prime}>n$ with $\bar{y}_{n}=\bar{y}_{n^{\prime}}$ (this implicitly implies that $n$ is associated to a grid point which does not belong to the last column). Moreover, formula $[A] \neg \psi_{=}^{1}$ imposes that $n^{\prime}$ must belong to the last column. Finally, it is required that there exists $0 \leq i \leq T$ s.t. $t_{i} \in \mathcal{V}([n, n]) \cap \mathcal{V}\left(\left[n^{\prime}, n^{\prime}\right]\right)$.

Notice that in the above definitions the use of the $\langle A\rangle$ operator enables us to deal with two key aspects:

1. we can predicate on all the intervals $\left[n, n^{\prime}\right]$ for any $n, n^{\prime} \in\{0, \ldots, N\}$, whereas, by using the $\langle B\rangle$ operator alone, we could predicate only on intervals of the form $[0, n]$;

2. we can predicate on the ending point of any current interval $\left[n, n^{\prime}\right]$, i.e., the interval $\left[n^{\prime}, n^{\prime}\right]$. Such a feature is missing in the logic $\mathrm{BD}_{\text {hom }}$ where we can predicate only on the beginning point of any current interval. For instance, the logic $\mathrm{BD}_{\text {hom }}$ cannot express properties like $\psi_{=}^{1}$ which checks whether the same set of propositional letters holds over the two ending points of an interval.

Let us define now the formula $\varphi_{\mathcal{T}}$ as $\varphi_{\mathcal{T}}=\psi_{\overline{t i l e}} \wedge \psi_{0, C} \wedge \psi_{\Rightarrow} \wedge \psi_{\Uparrow} \wedge \psi_{\text {prefix }}$. Since the models of $\psi_{\overline{t i l e}}$ represent all and only the possible finite tiling functions for $\mathcal{T}$ and $\psi_{0, C}, \psi_{\Rightarrow}, \psi_{\Uparrow}, \psi_{\text {prefix }}$ select the subset of such functions/models where conditions 1, 2, and 3, of Problem 1 together with condition 4 of Lemma 1 are fulfilled we can prove the following result.

Theorem 3. Given a instance $\mathcal{T}=(T, \Rightarrow, \Uparrow, C)$ of Problem $\square$ we have that $\mathcal{T}$ is a positive instance if and only if the $A B_{\text {hom }}$ formula $\varphi_{\mathcal{T}}$ is satisfiable over finite linear orders.

It is easy to see that the formula $\varphi_{\mathcal{T}}$ may be generated in LOGSPACE. It suffices to observe that we may define a multitape Turing Machine that performs the reduction using just a constant amount of working tapes each one holding either $\left\lceil\log _{2} T\right\rceil$ bits or $c$ bits. Finally, from such an observation together with Theorem 2 we obtain the main result of this section.

Theorem 4. The satisfiability problem for the logic $A B_{\text {hom }}$ over finite linear orders is EXPSPACE-hard.

We conclude this section by pointing out some interesting facts that allow us to better understand how the homogeneity assumption affects the satisfiability problem of the considered HS fragments. As a matter of fact the complexity of the satisfiability problem for $A_{h o m}$ over finite linear orders does not change if we consider its general version $A B$ (i.e., without homogeneity assumption [6]). On the contrary, the homogeneity assumption marks a deep difference in the fragment ABD. In fact, we shall prove in the next section that the satisfiabilty problem for $A B D_{\text {hom }}$ is decidable in exponential space whereas the problem for $A B D$ is known to be undecidable [11, 12]. As for model checking, the model checking problem over finite Kripke structures for $A_{h o m}$ is proved to be PSPACE-complete [3] while 
in this work we have already proved that the satisfiability problem over finite linear orders belongs to an higher complexity class (i.e., EXPSPACE). The tight complexity bound for the model checking problem over finite Kripke structures for $A B D_{h o m}$ is still open: we only know that for its three maximal proper fragments $\mathrm{AB}_{\text {hom }}, \mathrm{AD}_{\text {hom }}$ and $\mathrm{BD}_{\text {hom }}$ the model checkin problem is PSPACE-complete [3, 5].

\section{Homogeneous compass structures}

In this section, we introduce a spatial representation of homogeneous models, called homogeneous compass structures, which will be used to prove that the satisfiabilty problem for $\mathrm{ABD}_{\text {hom }}$ is decidable in exponential space in Section 5 .

Let $\varphi$ be a $\mathrm{BDA}_{\text {hom }}$ formula. We define the closure of $\varphi$, denoted by $\mathrm{Cl}(\varphi)$, as the set of all its sub-formulas and of their negations, plus formulas $\pi$ and $\neg \pi$. Moreover, we denote with $T F_{A}^{\varphi}=\{\psi$ : $\langle A\rangle \psi \in \mathrm{Cl}(\varphi)\}$ the set of all the arguments $\psi$ for $\langle A\rangle \psi$ formulas in $\mathrm{Cl}(\varphi)$. For every $\mathrm{BDA}_{\text {hom }}$ formula $\varphi$, it holds that $\mathrm{Cl}(\varphi) \leq 2|\varphi|+2$ and $\left|T F_{A}^{\varphi}\right| \leq|\mathrm{Cl}(\varphi)| / 2-1$.

A $\varphi$-atom (atom for short) is a pair $F_{\alpha}=(F, \alpha)$ where:

1. $F$ is a maximal subset of $\mathrm{Cl}(\varphi)$ that, for all $\psi \in \mathrm{Cl}(\varphi)$, satisfies the following three conditions: (i) $\psi \in F$ if and only if $\neg \psi \notin F$, (ii) if $\psi=\psi_{1} \vee \psi_{2}$, then $\psi \in F$ if and only if $\left\{\psi_{1}, \psi_{2}\right\} \cap F \neq \varnothing$, and (iii) if $\pi \in F$ then for every $[A] \psi \in F$ we have $\psi \in F$;

2. $\alpha$ is a function $\alpha: T F_{A}^{\varphi} \rightarrow\{\diamond, \diamond, \square\}$ that, for all $\psi \in T F_{A}^{\varphi}$, satisfies the following four conditions:

(i) if $\alpha(\psi)=\square$ then $\neg \psi \in F$; (ii) if $\psi \in F$ then $\alpha(\psi)=\diamond$; (iii) if $\pi \in F$ and $\alpha(\psi)=\diamond$ then

$\langle A\rangle \psi \in F$ and $\psi \notin F$; (iv) if $\pi \in F$ and $\alpha(\psi)=\diamond$ then $\psi \in F$.

For the sake of simplicity, from now on when we refer to $F_{\alpha}$ as a set, we refer to its first component $F$. For instance, when we write $\psi \in F_{\alpha}$, we mean $\psi \in F$. An atom $F_{\alpha}$ is final iff for every $\psi \in T F_{A}^{\varphi}$ we have $\alpha(\psi) \in\{\bullet, \square\}$. Let $\operatorname{At}(\varphi)$ be the set of all $\varphi$-atoms. We have that $|\operatorname{At}(\varphi)| \leq 2^{|\varphi|+1} \cdot 2^{|\varphi|-1}=2^{2|\varphi|}$, where $|\varphi|=|\mathrm{Cl}(\varphi)| / 2$.

For all $R \in\{A, B, D\}$, we introduce the functions $\operatorname{Req}_{R}, \mathrm{Obs}_{R}$, and $\mathrm{Box}_{R}$, that map each atom $F_{\alpha} \in$ $\operatorname{At}(\varphi)$ to the following subsets of $\mathrm{Cl}(\varphi)$ :

- $\operatorname{Req}_{R}\left(F_{\alpha}\right)=\{\psi \in \mathrm{Cl}(\varphi):\langle R\rangle \psi \in F\}$;

- $\operatorname{Obs}_{R}\left(F_{\alpha}\right)=\{\psi \in \mathrm{Cl}(\varphi):\langle R\rangle \psi \in \mathrm{Cl}(\varphi), \psi \in F\}$;

- $\operatorname{Box}_{R}\left(F_{\alpha}\right)=\{\psi \in \mathrm{Cl}(\varphi):[R] \psi \in F\}$.

Note that, for each $F_{\alpha} \in \operatorname{At}(\varphi)$ and each formula $\psi$, with $\psi \in\left\{\psi^{\prime}:\langle A\rangle \psi^{\prime} \in \operatorname{Cl}(\varphi)\right\}$, either $\psi \in \operatorname{Req}_{A}\left(F_{\alpha}\right)$ or $\neg \psi \in \operatorname{Box}_{A}\left(F_{\alpha}\right)$ and similarly for $B$ and $D$ (it implies that $\operatorname{Box}_{A}(\cdot), \operatorname{Box}_{B}(\cdot)$ and $\operatorname{Box}_{D}(\cdot)$ are not strictly necessary and are introduced only for technical convenience). By exploiting functions above, we define two binary relations $\rightarrow_{B}$ and $\rightarrow_{D}$ over $\operatorname{At}(\varphi)$ as follows. For all $F_{\alpha}, G_{\beta} \in \operatorname{At}(\varphi)$ we write

- $F_{\alpha} \rightarrow_{B} G_{\beta}$ iff $\operatorname{Req}_{B}\left(F_{\alpha}\right)=\operatorname{Req}_{B}\left(G_{\beta}\right) \cup \operatorname{Obs}_{B}\left(G_{\beta}\right)$ and for every $\psi \in T F_{A}^{\varphi}$ we have $\alpha(\psi)=\beta(\psi)$ if $\beta(\psi) \in\{\checkmark, \square\}$ or $\psi \notin F$;

- $F_{\alpha} \rightarrow{ }_{D} G_{\beta}$ iff $\operatorname{Req}_{D}\left(F_{\alpha}\right) \supseteq \operatorname{Req}_{D}\left(G_{\beta}\right) \cup \operatorname{Obs}_{D}\left(G_{\beta}\right)$.

Notice that from the definition of $\rightarrow_{B}$ (resp., $\rightarrow_{D}$ ), it easily follows that $\operatorname{Box}_{B}\left(F_{\alpha}\right) \subseteq G$ (resp., $\left.\operatorname{Box}_{D}\left(F_{\alpha}\right) \subseteq G\right)$. Notice also that $\rightarrow_{D}$ is transitive (by definition of atom, from $\operatorname{Req}_{R}\left(F_{\alpha}\right) \supseteq \operatorname{Req}_{R}\left(G_{\alpha}\right.$ ), it immediately follows that $\left.\operatorname{Box}_{R}\left(F_{\alpha}\right) \subseteq \operatorname{Box}_{R}\left(G_{\beta}\right)\right)$, while $\rightarrow_{B}$ is not.

Proposition 1. For each pair of atoms $F_{\alpha}, G_{\beta} \in \operatorname{At}(\varphi)$, we have that $F=G$ iff $\operatorname{Req}_{R}\left(F_{\alpha}\right)=\operatorname{Req}_{R}\left(G_{\beta}\right)$ for each $R \in\{A, B, D\}$, and $F \cap$ Prop $=G \cap$ Prop.

Given $N \in \mathbb{N}$, let $\mathbb{G}_{N}=\{(x, y): 0 \leq x \leq y \leq N\}$, given a formula $\varphi$, a $\varphi$-compass structure (compass structure, when $\varphi$ is clear from the context) is a pair $\mathcal{G}=(N, \mathcal{L})$, where $N \in \mathbb{N}$, and $\mathcal{L}: \mathbb{G}_{N} \rightarrow \operatorname{At}(\varphi)$ is a labelling function that satisfies the following properties: 
- (initial formula) $\varphi \in \mathcal{L}(0, N)$;

- $\left(A\right.$-consistency) for all $0 \leq x \leq y \leq N, \operatorname{Req}_{A}(\mathcal{L}(x, y))=\operatorname{Req}_{A}(\mathcal{L}(y, y))$;

- $\left(B\right.$-consistency) for all $0 \leq x \leq y<N, \mathcal{L}(x, y+1) \rightarrow_{B} \mathcal{L}(x, y)$; for all $0 \leq x \leq N, \operatorname{Req}_{B}(\mathcal{L}(x, x))=\varnothing$;

- $\left(D\right.$-consistency) for all $0 \leq x<x^{\prime} \leq y^{\prime}<y \leq N, \mathcal{L}(x, y) \rightarrow_{D} \mathcal{L}\left(x^{\prime}, y^{\prime}\right)$;

- (D-fulfilment) for all $0 \leq x \leq y \leq N$ and all $\psi \in \operatorname{Req}_{D}(\mathcal{L}(x, y))$, there exist $x<x^{\prime} \leq y^{\prime}<y$ such that $\psi \in \mathcal{L}\left(x^{\prime}, y^{\prime}\right)$

- (A-fulfilment) for every $0 \leq x \leq N$ atom $\mathcal{L}(x, N)$ is final.

Observe that the definition of $\rightarrow_{B}$ and $B$-consistency guarantee that all the existential requests via the relation $B$ (hereafter $B$-requests) are fulfilled in a compass structure.

We say that an atom $F \in \operatorname{At}(\varphi)$ is $B$-reflexive (resp., $D$-reflexive) if $F \rightarrow_{B} F$ (resp., $F \rightarrow_{D} F$ ). If $F$ is not $B$-reflexive (resp., $D$-reflexive), it is $B$-irreflexive (resp., $D$-irreflexive).

Let $\mathcal{G}=(N, \mathcal{L})$ be a compass structure. We define the function $\mathcal{P}: \mathbb{G}_{N} \rightarrow 2^{\text {Prop }}$ such that $\mathcal{P}(x, y)=$ $\left\{p \in\right.$ Prop $: p \in \mathcal{L}\left(x^{\prime}, x^{\prime}\right)$ for all $\left.x \leq x^{\prime} \leq y\right\}$. We say that a $\varphi$-compass structure $\mathcal{G}=\left(\mathbb{G}_{N}, \mathcal{L}\right)$ is homogeneous if for all $(x, y) \in \mathbb{G}_{N}, \mathcal{L}(x, y) \cap$ Prop $=\mathcal{P}(x, y)$. Hereafter, we will often write compass structure for homogeneous $\varphi$-compass structure.

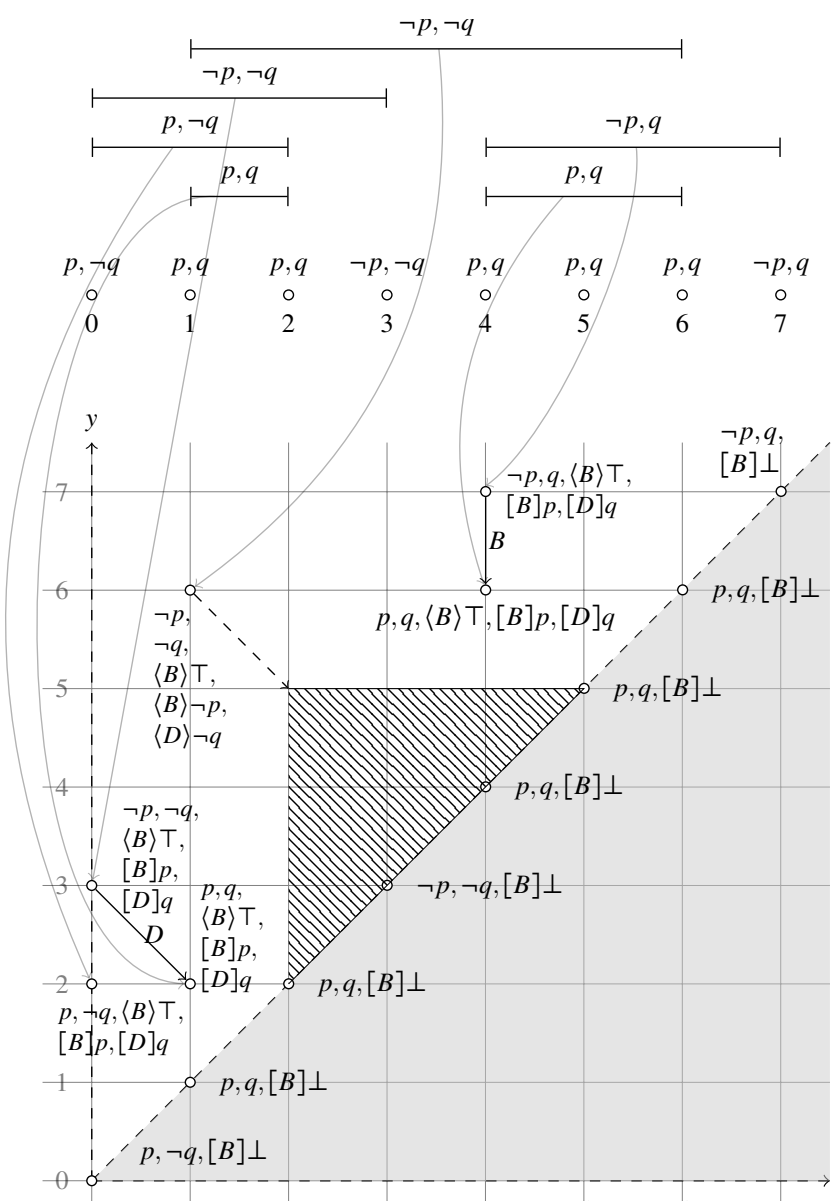

Figure 2: A homogeneous model and the corresponding compass structure.
Figure 2 depicts the homogeneous model $\mathbf{M}=(7, \mathcal{V})$ of Figure 1 $(a)$ with the corresponding compass structure $\mathcal{G}=(7, \mathcal{L})$, for a given formula $\varphi$. We assume that $\mathrm{Cl}(\varphi) \cap$ Prop $=$ $\{p, q\},\{\langle B\rangle \psi \in \mathrm{Cl}(\varphi)\}=\{\langle B\rangle \top,\langle B\rangle \neg p\}$, and $\{\langle D\rangle \psi \in \operatorname{Cl}(\varphi)\}=\{\langle D\rangle \neg q\}$. We know that, by the homogeneity assumption, the valuation of proposition letters at point-intervals determines that at non-point ones.

As an example, if an interval $[x, y]$ contains time point 3 , as, e.g., the interval $[1,6]$, then $\{p, q\} \cap \mathcal{V}([x, y])=\varnothing$. Similarly, if an interval $[x, y]$ contains time point 7 (resp., 0), then it must satisfy $\{p\} \cap \mathcal{V}([x, y])=\varnothing$ (resp., $\{q\} \cap \mathcal{V}([x, y])=\varnothing)$. As for the compass structure $\mathcal{G}$, we first observe that each interval $[x, y]$ in $\mathbf{M}$ is mapped to a point in the second octant of the $\mathbb{N} \times \mathbb{N}$ grid (in Figure 2, we depict the first quadrant of such a grid, where the first octant is shaded). Analogously, interval relations are mapped into special relations between points (by a slight abuse of terminology, we borrow the names of the interval relations). As an example, point $(0,2)$ begins $(0,3)$. Point $(1,6)$ has points $(2,2),(2,3),(3,3)$, $(2,4),(3,4),(4,4),(2,5),(3,5),(4,5),(5,5)$ as sub-intervals (see the hatched triangle). In general, all points $(x, x)$ are labelled with irreflexive atoms containing $[B] \perp$, while all points $(x, y)$, with $x<y$, are labelled with atoms containing $\langle B\rangle T$. The variety of atoms is exemplified by the 
following cases. Atom $\mathcal{L}(0,3)$ is both $B$-irreflexive and $D$-irreflexive, atom $\mathcal{L}(4,6)$ is both $B$-reflexive and $D$-reflexive, atom $\mathcal{L}(4,7)$ is $B$-irreflexive $\operatorname{(Box}_{B}(\mathcal{L}(4,7))=\{p\}$ and $\left.\neg p \in \mathcal{L}(4,7)\right)$ and $D$-reflexive $\left(\operatorname{Box}_{D}(\mathcal{L}(4,7))=\{q\}\right.$ and $\left.q \in \mathcal{L}(4,7)\right)$, and atom $\mathcal{L}(0,2)$ is $B$-reflexive $\left(\operatorname{Box}_{B}(\mathcal{L}(0,2))=\{p\}\right.$ and $p \in \mathcal{L}(0,2))$ and $D$-irreflexive $\left(\operatorname{Box}_{D}(\mathcal{L}(0,2))=\{q\}\right.$ and $\left.\neg q \in \mathcal{L}(0,2)\right)$. Finally, it holds that $\mathcal{L}(4,7) \rightarrow_{B}$ $\mathcal{L}(4,6)\left(\operatorname{Box}_{B}(\mathcal{L}(4,7))=\{p, q\}\right.$ and $\left.p, q \in \mathcal{L}(4,6)\right)$ and $\mathcal{L}(3,0) \rightarrow_{D} \mathcal{L}(1,2)\left(\operatorname{Box}_{D}(\mathcal{L}(3,0))=\{q\}\right.$ and $q \in \mathcal{L}(1,2))$.

The following theorem shows that compass structures are proper tools for solving the satisfiability problem (the proof is straightforward and thus omitted).

Theorem 5. A $\mathrm{ABD}_{\text {hom }}$ formula $\varphi$ is satisfiable iff there is a homogeneous $\varphi$-compass structure.

\section{The satisfiability problem for $\mathrm{ABD}_{\text {hom }}$ is decidable in EXPSPACE}

In this section, we show that the problem of checking whether a ABD formula $\varphi$ is satisfied by some homogeneous model can be decided in exponential space. We first prove that either $\varphi$ is unsatisfiable or it is satisfied by a model of at most doubly-exponential size in $|\varphi|$; then, we show that this model of doubly-exponential size can be guessed in single exponential space.

Theorem 6. Deciding whether a ABD formula $\varphi$ is satisfiable is a problem in EXPACE.

The proof consists of four main steps whose intuition will be detailed in the following (due to space bounds complete proofs are omitted).

Step 1: A finite characterisation of columns and of their relationships.

In this section, we first show that, in every compass structure, the atoms that appear in a column $x$ must respect a certain order, that is, they cannot be interleaved. Let $F_{\alpha}, G_{\beta}$, and $H_{\gamma}$ be three pairwise distinct atoms with $\operatorname{Req}_{A}\left(F_{\alpha}\right)=\operatorname{Req}_{A}\left(G_{\beta}\right)=H_{\gamma}$. In Figure 3. (a), we give a graphical account of the property to be proved, while, in Figure 3.(b), we show a violation (atom $H$ appears before and after atom $G$ moving upward along the column).

We preliminarily prove a fundamental property of $B$ irreflexive atoms.

Lemma 2. Let $\mathcal{G}=(N, \mathcal{L})$ be a compass structure. For all $x \leq y<N$, if $\operatorname{Req}_{B}(\mathcal{L}(x, y)) \subset \operatorname{Req}_{B}(\mathcal{L}(x, y+1))$, then $\mathcal{L}(x, y)$ is B-irreflexive.

Given atom $F_{\alpha}$ in a column, let us now provide a bound on the number of distinct atoms $G_{\beta}$ with $\operatorname{Req}_{A}\left(F_{\alpha}\right)=\operatorname{Req}_{A}\left(G_{\beta}\right)$ that can be placed above a $F_{\alpha}$ in a column, that takes into account $B$-requests, $D$-requests, negative literals in $F$, and the number of $\psi \in T F_{A}^{\varphi}$ such that $\alpha(\psi)=\diamond$.

Formally, we define a function $\Delta_{\uparrow}: \operatorname{At}(\varphi) \rightarrow \mathbb{N}$ as follows:

$$
\begin{aligned}
\Delta_{\uparrow}\left(F_{\alpha}\right)= & \left(2|\{\langle B\rangle \psi \in \operatorname{Cl}(\varphi)\}|-2\left|\operatorname{Req}_{B}\left(F_{\alpha}\right)\right|-\right. \\
& \left.\left|\operatorname{Obs}_{B}\left(F_{\alpha}\right) \backslash \operatorname{Req}_{B}\left(F_{\alpha}\right)\right|\right)+ \\
& \left(|\{\langle D\rangle \psi \in \operatorname{Cl}(\varphi)\}|-\left|\operatorname{Req}_{D}\left(F_{\alpha}\right)\right|\right)+ \\
& (|\{\neg p: p \in \operatorname{Cl}(\varphi) \cap \operatorname{Prop}\}|- \\
& \left.\left|\left\{\neg p: p \in \operatorname{Cl}(\varphi) \cap \operatorname{Prop} \wedge \neg p \in F_{\alpha}\right\}\right|\right)+ \\
& \left|\left\{\psi \in T F_{A}^{\varphi}: \alpha(\psi)=\diamond\right\}\right|
\end{aligned}
$$

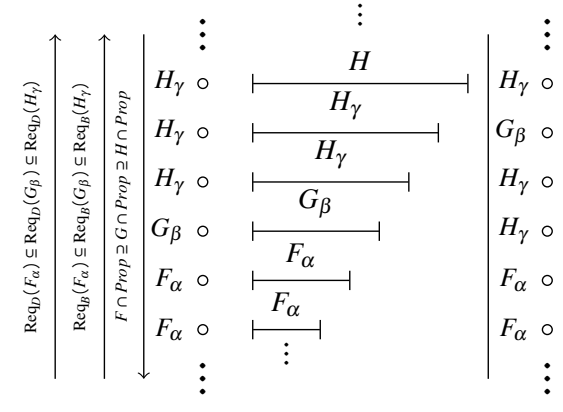

(a)

(b)

Figure 3: (a) Monotonicity of atoms along a column in a compass structure, together with a graphical account of the corresponding intervals and of how proposition letters and $B / D$ requests must behave. (b) An example of a violation of monotonicity. 
To understand why a factor 2 comes into play in the case of $B$-requestes, notice that to move down from an atom including $\langle B\rangle \psi$ to an atom including $\neg \psi,[B] \neg \psi$ one must pass through an atom including $\psi,[B] \neg \psi$. It can be easily checked that, for each $F_{\alpha} \in \operatorname{At}(\varphi), 0 \leq \Delta_{\uparrow}(F) \leq 5|\varphi|$.

To explain how $\Delta_{\uparrow}$ works, we give a simple example. Let $\{\psi:\langle B\rangle \psi \in \mathrm{Cl}(\varphi)\}=\left\{\psi_{1}\right\}$ and let $F_{\alpha} \rightarrow{ }_{B} G_{\beta} \rightarrow_{B} H_{\gamma}$, with $\operatorname{Req}_{B}\left(H_{\gamma}\right)=\left\{\psi_{1}\right\}$ and $\operatorname{Req}_{B}\left(G_{\beta}\right)=\operatorname{Req}_{B}\left(F_{\alpha}\right)=\varnothing$. For simplicity, let $T F_{A}^{\varphi}=\varnothing$, $\{\psi:\langle D\rangle \psi \in \operatorname{Cl}(\varphi)\}=\varnothing$, and thus $\operatorname{Req}_{D}\left(H_{\gamma}\right)=\operatorname{Req}_{D}\left(G_{\beta}\right)=\operatorname{Req}_{D}\left(F_{\alpha}\right)=\varnothing$, and $\left(H_{\gamma} \cap G_{\beta} \cap F_{\alpha}\right) \cap$ Prop $=$ Prop $=\{p\}$. It holds that $\Delta_{\uparrow}\left(F_{\alpha}\right)=(2 \cdot 1-2 \cdot 0-0)+(0-0)+(1-0)+0=3, \Delta_{\uparrow}\left(G_{\beta}\right)=$ $(2 \cdot 1-2 \cdot 0-1)+(0-0)+(1-0)+0=2$, and $\Delta_{\uparrow}\left(H_{\gamma}\right)=(2 \cdot 1-2 \cdot 1-0)+(0-0)+(1-0)+0=1$.

We say that an atom $F$ is initial if and only if $\pi \in F_{\alpha}$. A B-sequence is a sequence of atoms $\mathrm{Sh}_{B}=$ $F_{\alpha_{0}}^{0} \ldots F_{\alpha_{n}}^{n}$ such that: $1 . F_{\alpha_{0}}^{0}$ is initial and $F_{\alpha_{n}}^{n}$ is final; 2 . for all $0<i \leq n$ we have $F_{\alpha_{i}}^{i} \rightarrow_{B} F_{\alpha_{i-1}}^{i-1}, \operatorname{Req}_{D}\left(F_{i}\right) \supseteq$ $\operatorname{Req}_{D}\left(F_{i-1}\right)$, and $F_{i} \cap$ Prop $\subseteq F_{i-1} \cap$ Prop.

A $B$-sequence $\mathrm{Sh}_{B}=F_{\alpha_{0}}^{0} \ldots F_{\alpha_{n}}^{n}$ is minimal iff for every $0 \leq i<n$ then for every $0 \leq i<n \Delta_{\uparrow}\left(F_{\alpha_{i}}^{i}\right)>$ $\Delta_{\uparrow}\left(F_{\alpha_{i+1}}^{i+1}\right)$. Let us observe that for every minimal $B$-sequence $\operatorname{Sh}_{B}=F_{\alpha_{0}}^{0} \ldots F_{\alpha_{n}}^{n}$ we have $n \leq 5|\varphi|$ (i.e., the length of a minimal $B$-sequence is at most $5|\varphi|+1)$.

Let $\mathcal{G}=(N, \mathcal{L})$ be a compass structure for $\varphi$ and $0 \leq x \leq N$. We define the shading of $x$ in $\mathcal{G}$, written $\operatorname{Sh}^{\mathcal{G}}(x)$, as the sequence of pairs atoms $\left(\mathcal{L}\left(x, y_{0}\right), y_{0}\right) \ldots\left(\mathcal{L}\left(x, y_{m}\right), y_{m}\right)$ such that:

1. $y_{i}<y_{i+1}$ for every $0 \leq i<m$;

2. $\left\{\Delta_{\uparrow}(\mathcal{L}(x, y)): 0 \leq y \leq N\right\}=\left\{\Delta_{\uparrow}\left(\mathcal{L}\left(x, y_{i}\right)\right): 0 \leq i \leq m\right\}$;

3. for every $0 \leq i \leq m$ we have $y_{i}=\min \left\{0 \leq y \leq N: \Delta_{\uparrow}\left(\mathcal{L}\left(x, y_{i}\right)\right)=\Delta_{\uparrow}(\mathcal{L}(x, y))\right\}$, i.e., $y_{i}$ is the minimum height on the column $x$ that exhibits its value for $\Delta_{\uparrow}$.

For every $0 \leq x \leq N$ let $\operatorname{Sh}^{\mathcal{G}}(x)=\mathcal{L}\left(x, y_{0}\right) \ldots \mathcal{L}\left(x, y_{m}\right)$ we denote with $\operatorname{Sh}_{B}^{\mathcal{G}}(x)$ the sequence of atoms $\mathcal{L}\left(x, y_{0}\right) \ldots \mathcal{L}\left(x, y_{m}\right)$, and with $\mathrm{Sh}_{\mathbb{N}}^{\mathcal{G}}(x)$ the sequence of natural numbers $y_{0} \ldots y_{m}$, that is, the projections of $\operatorname{Sh}^{\mathcal{G}}(x)$ of on the first and the second components of its elements, respectively.

The next lemma easily follows from the definitions of $B$-sequence and shading (the proof is omitted).

Lemma 3. Let $\mathcal{G}=(N, \mathcal{L})$ be a compass structure and $0 \leq x \leq N$, then $\operatorname{Sh}_{B}^{\mathcal{G}}(x)$ is a minimal B-sequence.

Step 2: Spatial arrangement of atoms between columns.

By exploiting the above (finite) characterisation of columns, we can define a natural equivalence relation of finite index over columns: we say that two columns $x, x^{\prime}$ are equivalent, written $x \sim x^{\prime}$, if and only if $\operatorname{Sh}_{B}^{\mathcal{G}}(x)=\operatorname{Sh}_{B}^{\mathcal{G}}\left(x^{\prime}\right)$.

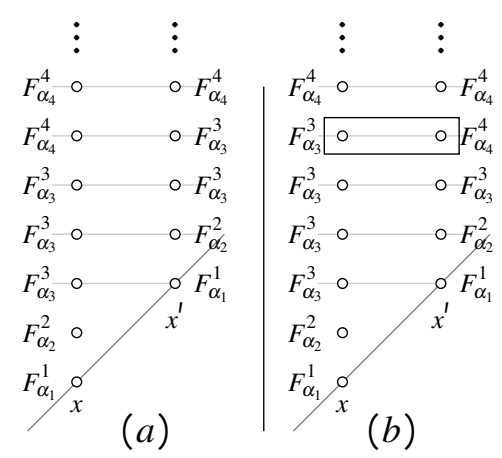

Figure 4: Two equivalent columns that respect the order $(a)$ and two equivalent columns that violates it $(b)$.
In the following, we prove that equivalent columns can be totally ordered according to a given partial order relation over their shadings. Formally, for any two equivalent columns $x \sim$ $x^{\prime}$ let $\operatorname{Sh}^{\mathcal{G}}(x)=\left(\mathcal{L}\left(x, y_{0}\right), y_{0}\right) \ldots\left(\mathcal{L}\left(x, y_{m}\right), y_{m}\right)$ and $\operatorname{Sh}^{\mathcal{G}}\left(x^{\prime}\right)=$ $\left(\mathcal{L}\left(x, \bar{y}_{0}\right), \bar{y}_{0}\right) \ldots\left(\mathcal{L}\left(x, \bar{y}_{m}\right), \bar{y}_{m}\right), \mathrm{Sh}^{\mathcal{G}}(x) \leq \operatorname{Sh}^{\mathcal{G}}\left(x^{\prime}\right)$ if and only if for every $0 \leq i \leq m$ we have $y_{i} \leq \bar{y}_{i}$. Intuitively, if we recall that, by definition, for every $0 \leq i \leq m$ the row $y_{i}$ (resp. $\bar{y}_{i}$ ) is the the minimum row for which atom $\mathcal{L}\left(x, y_{i}\right)=\mathcal{L}\left(x^{\prime}, \bar{y}_{i}\right)$ (from $\left.\operatorname{Sh}_{B}^{\mathcal{G}}(x)=\operatorname{Sh}_{B}^{\mathcal{G}}\left(x^{\prime}\right)\right)$ occurs on column $x$ (resp. $x^{\prime}$ ) meaning that, moving upward column $x^{\prime}$, an atom cannot appear until it has appeared on column $x$. In Fig. 4(a), we depict two equivalent columns that satisfy such a property. In general, when moving upward, atoms on $x^{\prime}$ are often "delayed" with respect to atoms in $x$, the limit case being when atoms on the same row are equal. 
In Fig. 4(b), a violation of the property (boxed atoms) is shown. The following lemma shows that such a violation never occurs in a compass structure.

Lemma 4. Let $\mathcal{G}=(N, \mathcal{L})$ be a compass structure. For every pair of equivalent columns $x \sim x^{\prime}$ with $0 \leq x<x^{\prime} \leq N$, it holds that $\operatorname{Sh}^{\mathcal{G}}(x)<\operatorname{Sh}^{\mathcal{G}}\left(x^{\prime}\right)$.

Step 3: $B$-sequence suffixes starting at the same row have bounded variability in $|\varphi|$.

Let us now provide a very strong characterization of the rows in a compass structure by making use of a covering property, depicted in Fig. 5.

Let $\mathcal{G}=(N, \mathcal{L})$ be a compass structure and let $0 \leq x \leq y$. We define $\mathbb{S}_{\rightarrow}(x, y)$ as the set $\left\{\left(\operatorname{Sh}_{B}^{\mathcal{G}}\left(x^{\prime}\right), \mathcal{L}\left(x^{\prime}, y\right)\right): x^{\prime}>\right.$ $x\} . \mathbb{S}_{\rightarrow}(x, y)$ collects the equivalence classes of $\sim$ which are witnessed to the right of $x$ on row $y$ by means of their B-sequence plus a "pointer" to the "current atom", that is, the atoms they are exposing on $y$. If $\mathcal{G}=(N, \mathcal{L})$ is homogeneous (as in our setting), for all $0 \leq x \leq y \leq$ $N$, the number of possible sets $\mathbb{S}_{\rightarrow}(x, y)$ is bounded by $2^{6^{5|\varphi|^{2}+2|\varphi|} \cdot \frac{2}{3}^{5|\varphi|+2}}$, that is, it is doubly exponential in the size of $|\varphi|$. For every $0 \leq x \leq y \leq N$ let us define the fingerprint of $(x, y)$ in $\mathcal{G}$, written $f p_{\mathcal{G}}(x, y)$, as the triple $f p_{\mathcal{G}}(x, y)=$ $\left(\operatorname{Sh}_{B}^{\mathcal{G}}(x), \mathcal{L}(x, y), \mathbb{S}_{\rightarrow}(x, y)\right)$. Lemma 5 constrains the way in which two columns $x, x^{\prime}$, with $x<x^{\prime}$ and $x \sim x^{\prime}$, evolve from a given row $y$ on when $f p_{\mathcal{G}}(x, y)=f p_{\mathcal{G}}\left(x^{\prime}, y\right)$.

For two atoms $F_{\alpha}$ and $G_{\beta}$, we say that they are equivalent

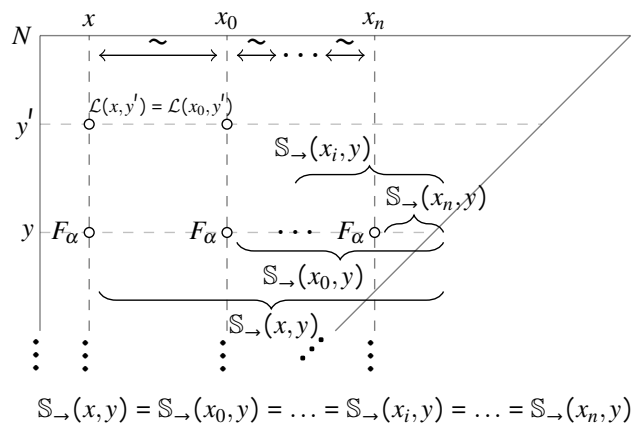

Figure 5: A graphical account of the behaviour of covered points. We have that $x$ is covered by $x_{0}<\ldots<x_{n}$ on row $y$ and thus the labelling of points on column $x$ above $(x, y)$ is exactly the same of the correspondent points on column $x_{0}$ above $\left(x_{0}, y\right)$, that is, $\mathcal{L}\left(x, y^{\prime}\right)=\mathcal{L}\left(x_{0}, y^{\prime}\right)$, for all $y \leq y^{\prime} \leq N$. modulo $A$, written $F_{\alpha} \equiv_{\neg A} G_{\beta}$ if and only if $F \backslash \operatorname{Req}_{A}\left(F_{\alpha}\right)=$ $G \backslash \operatorname{Req}_{A}\left(G_{\beta}\right)$ and $\alpha=\beta$ (i.e., $F_{\alpha}$ and $G_{\beta}$ have at most different $\langle A\rangle$ requests).

Lemma 5. Let $\mathcal{G}=(N, \mathcal{L})$ be a compass structure and let $0 \leq x<x^{\prime} \leq y \leq N$. If $f p_{\mathcal{G}}(x, y)=f p_{\mathcal{G}}\left(x^{\prime}, y\right)$ and $y^{\prime}$ is the smallest point greater than $y$ such that $\mathcal{L}\left(x, y^{\prime}\right) \neq_{\neg A} \mathcal{L}(x, y)$, if any, and $N$ otherwise, then, for all $y \leq y^{\prime \prime} \leq y^{\prime}, \mathcal{L}\left(x, y^{\prime \prime}\right)=\mathcal{L}\left(x^{\prime}, y^{\prime \prime}\right)$.

From Lemma 5 , the next corollary follows.

Corollary 1. Let $\mathcal{G}=(N, \mathcal{L})$ be a compass structure and let $0 \leq x<x^{\prime} \leq y \leq N$. If $f p_{\mathcal{G}}(x, y)=f p_{\mathcal{G}}\left(x^{\prime}, y\right)$ and $y^{\prime}$ is the smallest point greater than $y$ such that $\mathcal{L}\left(x, y^{\prime}\right) \neq_{\neg A} \mathcal{L}(x, y)$, if any, and $N$ otherwise, then, for every pair of points $\bar{x}, \bar{x}^{\prime}$, with $x<\bar{x}<x^{\prime}<\bar{x}^{\prime}$, with $\mathcal{L}(\bar{x}, y)=\mathcal{L}\left(\bar{x}^{\prime}, y\right)$ and $\bar{x} \sim \bar{x}^{\prime}+x$, it holds that $\mathcal{L}\left(\bar{x}, y^{\prime \prime}\right)=\mathcal{L}\left(\bar{x}^{\prime}, y^{\prime \prime}\right)$, for all $y \leq y^{\prime \prime} \leq y^{\prime}$.

The above results lead us to the identification of those points $(x, y)$ whose behaviour perfectly reproduces that of a number of points $\left(x^{\prime}, y\right)$ on their right with $f p_{\mathcal{G}}(x, y)=f p_{\mathcal{G}}\left(x^{\prime}, y\right)$. These points $(x, y)$, like all points "above" them, are irrelevant with respect to fulfilment in a compass structure. We call them covered points.

Definition 1. Let $\mathcal{G}=(N, \mathcal{L})$ be a compass structure and $0 \leq x \leq y \leq N$. We say that $(x, y)$ is covered iff there exist $n+1=\Delta_{\uparrow}(\mathcal{L}(x, y))$ distinct points $x_{0}<\ldots<x_{n} \leq y$, with $x<x_{0}$, such that for all $0 \leq i \leq n$, $f p_{\mathcal{G}}(x, y)=f p_{\mathcal{G}}\left(x_{i}, y\right)$. In such a case, we say that $x$ is covered by $x_{0}<\ldots<x_{n}$ on $y$.

Lemma 6. Let $\mathcal{G}=(N, \mathcal{L})$ be a compass structure and let $x, y$, with $0 \leq x \leq y \leq N$, be two points such that $x$ is covered by points $x_{0}<\ldots<x_{n}$ on $y$. Then, for all $y \leq y^{\prime} \leq N$, it holds that $\mathcal{L}\left(x, y^{\prime}\right)=\mathcal{L}\left(x_{0}, y^{\prime}\right)$. 


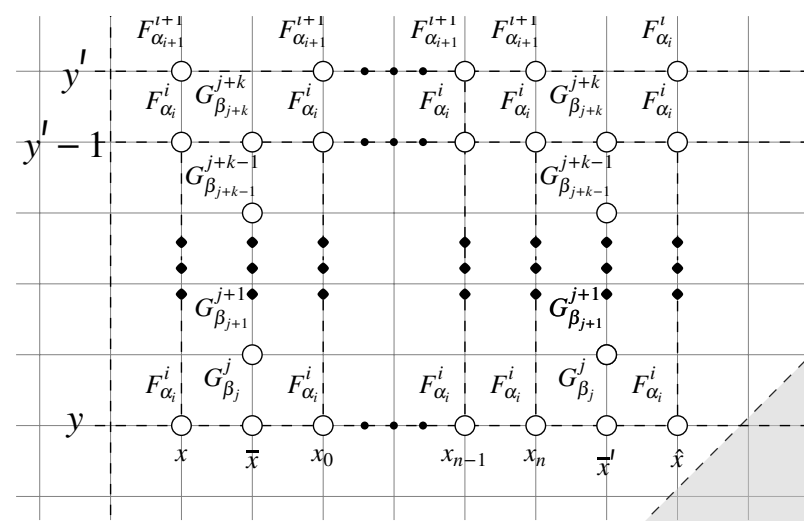

Figure 6: An intuitive account of the statement of Lemma6.
In Figure 6, we give an intuitive account of the notion of covered points and of the statement of Lemma 6. First of all, we observe that, since $\mathbb{S}_{\rightarrow}(x, y)=\mathbb{S}_{\rightarrow}\left(x_{0}, y\right)=\ldots=\mathbb{S}_{\rightarrow}\left(x_{n}, y\right)$ and, for all $0 \leq j, j^{\prime} \leq n$, it holds that $\left(\operatorname{Sh}_{B}^{\mathcal{G}}\left(x_{j}\right), \mathcal{L}\left(x_{j}, y\right)\right)=$ $\left(\operatorname{Sh}_{B}^{\mathcal{G}}\left(x_{j^{\prime}}\right), \mathcal{L}\left(x_{j^{\prime}}, y\right)\right)$, there exists $x_{n}<\hat{x} \leq y$ such that $\left(\operatorname{Sh}_{B}^{\mathcal{G}}\left(x_{n}\right), \mathcal{L}\left(x_{n}, y\right)\right)=\left(\operatorname{Sh}_{B}^{\mathcal{G}}(\hat{x}), \mathcal{L}(\hat{x}, y)\right)$, and $\hat{x}$ is the smallest point greater than $x_{n}$ that satisfies such a condition. Now, it may happen that $\mathbb{S}_{\rightarrow}\left(x_{n}, y\right) \supset \mathbb{S}_{\rightarrow}(\hat{x}, y)$, and all points $\bar{x}^{\prime}>x_{n}$ with $\left(\operatorname{Sh}_{B}^{\mathcal{G}}\left(\bar{x}^{\prime}\right), \mathcal{L}\left(\bar{x}^{\prime}, y\right)\right)=\left(\operatorname{Sh}_{B}^{\mathcal{G}}(\bar{x}), \mathcal{L}(\bar{x}, y)\right)$, for some $x<\bar{x}<x_{n}$, are such that $x_{n}<\bar{x}^{\prime}<\hat{x}$. Then, it can be the case that, for all $0 \leq i \leq n, \mathcal{L}\left(x_{i}, y^{\prime}\right)=F_{\alpha_{i+1}}^{i+1}$, as all points $\left(x_{i}, y^{\prime}\right)$ satisfy some $D$-request $\psi$ that only belongs to $\mathcal{L}\left(\bar{x}^{\prime}, y^{\prime}-1\right)$. In such a case, as shown in Figure 6, $\mathcal{L}\left(\hat{x}, y^{\prime}\right)=F_{\alpha_{i}}^{i}$, because for all points $\left(\hat{x}^{\prime}, \hat{y}^{\prime}\right)$, with $\hat{x}<\hat{x}^{\prime} \leq \hat{y}^{\prime}<y^{\prime}, \psi \notin \mathcal{L}\left(\hat{x}^{\prime}, \hat{y}^{\prime}\right)$. Hence, $\left(\operatorname{Sh}_{B}^{\mathcal{G}}\left(x_{n}\right), F_{\alpha_{i+1}}^{i+1}\right) \in \mathbb{S}_{\rightarrow}\left(x_{j}, y^{\prime}\right)$ for all $0 \leq j<n$, but $\left(\operatorname{Sh}_{B}^{\mathcal{G}}\left(x_{n}\right), F_{\alpha_{i+1}}^{i+1}\right) \notin \mathbb{S}_{\rightarrow}\left(x_{n}, y^{\prime}\right)$. Then, by applying Corollary 1, we have that $\mathbb{S}_{\rightarrow}\left(x_{0}, y^{\prime}\right)=\mathbb{S}_{\rightarrow}\left(x_{n-1}, y^{\prime}\right)$. Since $\Delta_{\uparrow}\left(F_{\alpha_{i+1}}^{i+1}\right)<\Delta_{\uparrow}\left(F_{\alpha_{i}}^{i}\right)(=n)$, it holds that $\Delta_{\uparrow}\left(F_{\alpha_{i+1}}^{i+1}\right) \leq n-1$. The same argument can then be applied to $x, x_{0}, \ldots, x_{n-1}$ on $y^{\prime}$, and so on.

Step 4: A contraction method for homogeneous compass structures.

Let us now complete the proof of Theorem 6 by providing a small model theorem for compass structures. By exploiting Lemma 6, we can show that, for each row $y$, the cardinality of the set of columns $x_{1}, \ldots, x_{m}$ which are not covered on $y$ is exponential in $|\varphi|$. Then, the sequence of triplets for non-covered points that appear on $y$ is bounded by an exponential value on $|\varphi|$.

It follows that, in a compass structure of size more than doubly exponential in $|\varphi|$, there exist two rows $y, y^{\prime}$, with $y<y^{\prime}$, such that the sequences of the triplets for non-covered points that appear on $y$ and $y^{\prime}$ are exactly the same. This allows us to apply a "contraction" between $y$ and $y^{\prime}$ on the compass structure. An example of how contraction works is given in Figure 7

First of all, notice that rows 7 and 11 feature the same sequences for triplets of non-covered points, and that, on any row, each covered point is connected by an edge to the non-covered point that "behaves" in the same way. More precisely, we have that column 2 behaves as column 4 between $y=7$ and $y^{\prime}=15$, columns 3,5 , and 7 be-

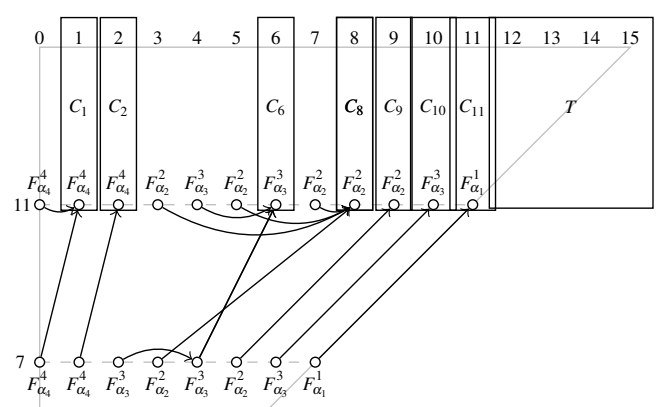

Figure 7: An example of contraction, where compass structure (a) is contracted into compass structure (b).

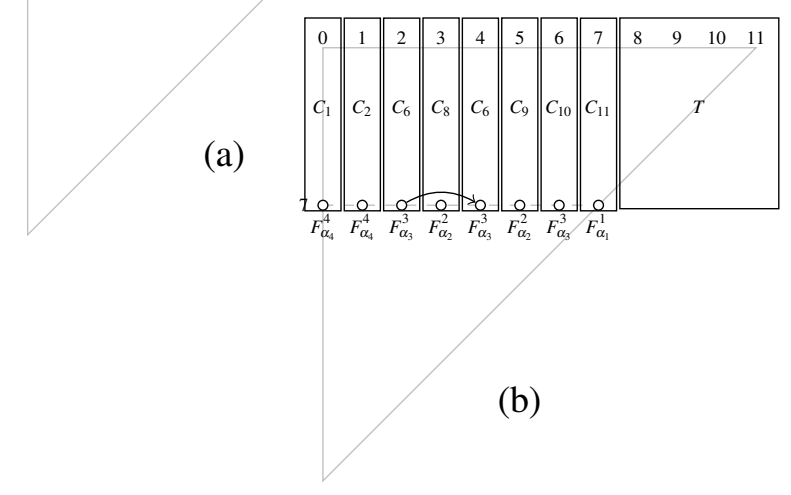


have as column 8 between $y=11$ and $y^{\prime}=15$, and column 4 behaves as column 6 between $y=11$ and $y^{\prime}=15$. The compass structure in Figure 7(a) can thus be shrinked into the compass structure in Figure 7.(b), where each column of non-covered points $x$ on $y^{\prime}$ is copied above the corresponding non-covered point $x^{\prime}$ on $y$. Moreover, the column of a non-covered point $x$ on $y^{\prime}$ is copied over all the points which are covered by the non-covered point $x^{\prime}$ corresponding to $x$ on $y$. This is the case with point 2 in Figure 7.(b) which takes the new column of its "covering" point 4 . The resulting compass structure is $y^{\prime}-y$ shorter than the original one, and we can repeatedly apply the contraction step until we achieve the desired bound.

The next corollary, which easily follows from Lemma 6 is crucial for the proof of the EXPSPACE membership of the satisfiability problem for $\mathrm{BDA}_{h o m}$. Roughly speaking, it states that the property of "being covered" propagates upward.

Corollary 2. Let $\mathcal{G}=(N, \mathcal{L})$ be a compass structure. Then, for every covered point $(x, y)$, it holds that, for all $y \leq y^{\prime} \leq N$, point $\left(x, y^{\prime}\right)$ is covered as well.

From Corollary 2, it immediately follows that, for every covered point $(x, y)$ and every $y \leq y^{\prime} \leq N$, there exists $x^{\prime}>x$ such that $\mathcal{L}\left(x^{\prime}, y^{\prime}\right)=\mathcal{L}\left(x, y^{\prime}\right)$. Hence, for all $\bar{x}, \bar{y}$, with $\bar{x}<x \leq y^{\prime}<\bar{y}$, and any $D$-request $\psi \in \operatorname{Req}_{D}(\mathcal{L}(\bar{x}, \bar{y})) \cap \operatorname{Obs}_{D}(\mathcal{L}(x, y))$, we have that $\psi \in \mathcal{L}\left(x^{\prime}, y\right)$, with $x^{\prime}>x$. This allows us to conclude that if $(x, y)$ is covered, then all points $\left(x, y^{\prime}\right)$, with $y^{\prime} \geq y$, are irrelevant from the point of view of $D$ requests. Let $\mathcal{G}=(N, \mathcal{L})$ be a compass structure and $0 \leq y \leq N$. We define the set of witnesses of $y$ as the set $\operatorname{Wit}_{\mathcal{G}}(y)=\{x:(x, y)$ is not covered $\}$. Corollary 2 guarantees that, for any row $y$, the shading $\operatorname{Sh}_{B}^{\mathcal{G}}(x)$ and the labelling $\mathcal{L}(x, y)$ of witnesses $x \in \mathrm{Wit}_{\mathcal{G}}(y)$ are sufficient, bounded, and unambiguous pieces of information that one needs to maintain about $y$.

Given a compass structure $\mathcal{G}=(N, \mathcal{L})$ and $0 \leq y \leq N$, we define the row blueprint of $y$ in $\mathcal{G}$, written $\operatorname{Row}_{\mathcal{G}}(y)$, as the sequence $\operatorname{Row}_{\mathcal{G}}(y)=\left(\operatorname{Sh}_{B}^{0}, F_{\alpha_{0}}^{0}\right) \ldots\left(\operatorname{Sh}_{B}^{m}, F_{\alpha_{m}}^{m}\right)$ such that $m+1=\left|\operatorname{Wit}_{\mathcal{G}}(y)\right|$ and there exists a bijection $b: \operatorname{Wit}_{\mathcal{G}}(y) \rightarrow\{0, \ldots, m\}$ such that, for every $x \in \operatorname{Wit}_{\mathcal{G}}(y)$, it holds that $\operatorname{Sh}_{B}^{\mathcal{G}}(x)=\operatorname{Sh}_{B}^{b(x)}$ and $\mathcal{L}(x, y)=F_{\alpha_{b(x)}}^{b(x)}$, and for every $x, x^{\prime}$ in $\operatorname{Wit}_{\mathcal{G}}(y), b(x)<b\left(x^{\prime}\right) \leftrightarrow x<x^{\prime}$. Now, we are ready to prove the following small model theorem.

Theorem 7. Let $\mathcal{G}=(N, \mathcal{L})$ be a compass structure. If there exist two points $y, y^{\prime}$, with $0 \leq y<y^{\prime} \leq N$, such that $\operatorname{Row}_{\mathcal{G}}(y)=\operatorname{Row}_{\mathcal{G}}\left(y^{\prime}\right)$, then there exists a compass structure $\mathcal{G}^{\prime}=\left(N^{\prime}, \mathcal{L}^{\prime}\right)$ with $N^{\prime}=N-\left(y^{\prime}-y\right)$.

The proof of Theorem 6 , is completed by proving that if a $\mathrm{BDA}_{h o m}$ formula is satisfiable, then it is satisfied by a doubly exponential compass structure, whose existence can be checked in exponential space.

Theorem 8. Let $\varphi$ be a BD formula. It holds that $\varphi$ is satisfiable iff there is a compass structure $\mathcal{G}=(N, \mathcal{L})$ for it such that $N \leq 2^{5|\varphi| \cdot\left(6^{10|\varphi|^{2}+4|\varphi|} \cdot 2^{10|\varphi|+4}\right)}$, whose existence can be checked in EXPSPACE.

\section{Conclusions}

In this paper, we prove that the satisfiability problem for $\mathrm{ABD}_{\text {hom }}$ over finite homogeneous linear orders is EXPSPACE-complete. This result stems a number of observations regarding the complexity landscape of the satisfiability and model checking problems related to HS interpreted over homogeneous structures $\left(\mathrm{HS}_{\text {hom }}\right)$ : 1. it improves the previously-known non-elementary upper bound [13]; 2. it provides a first EXPSPACE-complete fragment of $\mathrm{HS}_{\text {hom }}$ w.r.t. to the satisfiability problem [3].

A more important fact regards how the results for $\mathrm{ABD}_{\text {hom }}$ can enlight the problem of determining the exact complexity of the satisfiability problem for the fragment $\mathrm{BE}_{\text {hom }}$ which is still open today. As 
a matter of fact $\mathrm{ABD}_{\text {hom }}$ and $\mathrm{BE}_{\text {hom }}$ are not comparable from an expressive standpoint [6]. However, by means of $\mathrm{ABD}_{\text {hom }}$ we can capture a fragment of $\mathrm{BE}_{\text {hom }}$ that is $\mathrm{BD}_{\text {hom }}$ plus a restricted version of the $\langle E\rangle$ operator namely $\langle E\rangle_{\pi} \psi=\langle A\rangle(\pi \wedge \psi)$ that allows one to predicate on the ending point of an interval. As we show in Section 3 , this is the only key property that enables the jump in complexity from $\mathrm{BD}_{\text {hom }}$ (PSPACE-Complete) to $\mathrm{ABD}_{\text {hom }}$ (EXPSPACE-Complete) w.r.t. the satisfiability problem. It is easy to see that the result presented here can be easily extended to the case of homogeneous structures isomorphic to $\mathbb{N}$.

In the future we plan to consider the satisfiability/model checking problem of (fragments of) $\mathrm{HS}_{\text {hom }}$ interpreted over linear order like $\mathbb{Q}$ and $\mathbb{R}$. Finally, let us point out that the precise characterization of the complexity of the satisfiability problem for $\mathrm{BE}_{\text {hom }}$ over finite structures is still the main open problem on the path of determining the complexity of the satisfiability problem for $\mathrm{HS}_{\text {hom }}$.

\section{References}

[1] Laura Bozzelli, Alberto Molinari, Angelo Montanari, Adriano Peron \& Pietro Sala (2017): Satisfiability and Model Checking for the Logic of Sub-Intervals under the Homogeneity Assumption. In Ioannis Chatzigiannakis, Piotr Indyk, Fabian Kuhn \& Anca Muscholl, editors: 44th International Colloquium on Automata, Languages, and Programming, ICALP 2017, July 10-14, 2017, Warsaw, Poland, LIPIcs 80, Schloss Dagstuhl - Leibniz-Zentrum fuer Informatik, pp. 120:1-120:14, doi:10.4230/LIPIcs . ICALP . 2017.120.

[2] Laura Bozzelli, Alberto Molinari, Angelo Montanari, Adriano Peron \& Pietro Sala (2019): Interval vs. Point Temporal Logic Model Checking: An Expressiveness Comparison. ACM Trans. Comput. Log. 20(1), pp. 4:1-4:31, doi:10.1305/ndjf1/1093635589.

[3] Laura Bozzelli, Alberto Molinari, Angelo Montanari, Adriano Peron \& Pietro Sala (2019): Which fragments of the interval temporal logic HS are tractable in model checking? Theor. Comput. Sci. 764, pp. 125-144, doi:10.1016/j.tcs.2018.04.011.

[4] Laura Bozzelli, Angelo Montanari, Adriano Peron \& Pietro Sala (2020): On a Temporal Logic of Prefixes and Infixes. In Javier Esparza \& Daniel Král', editors: 45th International Symposium on Mathematical Foundations of Computer Science, MFCS 2020, August 24-28, 2020, Prague, Czech Republic, LIPIcs 170, Schloss Dagstuhl - Leibniz-Zentrum für Informatik, pp. 21:1-21:14, doi:10.4230/LIP Ics .MFCS .2020 . 21.

[5] Laura Bozzelli, Angelo Montanari, Adriano Peron \& Pietro Sala (2021): Pspace-completeness of the temporal logic of sub-intervals and suffixes. To appear in: Carlo Combi, Johan Eder, and Mark Reynolds eds. proceedings of 28th International Symposium on Temporal Representation and Reasoning, TIME 2021, September 27-29, 2021, Klagenfurt, Austria, LIPIcs, Schloss Dagstuhl - Leibniz-Zentrum für Informatik.

[6] Davide Bresolin, Dario Della Monica, Angelo Montanari, Pietro Sala \& Guido Sciavicco (2014): Interval temporal logics over strongly discrete linear orders: Expressiveness and complexity. Theor. Comput. Sci. 560, pp. 269-291, doi:10.1016/j.tcs.2014.03.033.

[7] Davide Bresolin, Angelo Montanari, Pietro Sala \& Guido Sciavicco (2011): Optimal Tableau Systems for Propositional Neighborhood Logic over All, Dense, and Discrete Linear Orders. In Kai Brünnler \& George Metcalfe, editors: Automated Reasoning with Analytic Tableaux and Related Methods - 20th International Conference, TABLEAUX 2011, Bern, Switzerland, July 4-8, 2011. Proceedings, Lecture Notes in Computer Science 6793, Springer, pp. 73-87, doi:10.1007/978-3-642-22119-4_8.

[8] Peter van Emde Boas (1997): The convenience of tilings. CRC Press.

[9] Valentin Goranko, Angelo Montanari \& Guido Sciavicco (2004): A Road Map of Interval Temporal Logics and Duration Calculi. Journal of Applied Non-Classical Logics 14(1-2), pp. 9-54, doi:10.3166/jancl . 14.9-54.

[10] Joseph Y. Halpern \& Yoav Shoham (1991): A Propositional Modal Logic of Time Intervals. Journal of ACM 38(4), pp. 935-962, doi:10.1145/115234.115351. 
[11] Jerzy Marcinkowski \& Jakub Michaliszyn (2014): The Undecidability of the Logic of Subintervals. Fundam. Inform. 131(2), pp. 217-240, doi:10.3233/FI-2014-1011.

[12] Jerzy Marcinkowski, Jakub Michaliszyn \& Emanuel Kieronski (2010): B and D Are Enough to Make the Halpern-Shoham Logic Undecidable. In Samson Abramsky, Cyril Gavoille, Claude Kirchner, Friedhelm Meyer auf der Heide \& Paul G. Spirakis, editors: Automata, Languages and Programming, 37th International Colloquium, ICALP, Bordeaux, France, July 6-10, Proceedings, Part II, LNCS 6199, Springer, pp. 357-368, doi:10.1007/978-3-642-14162-1_30.

[13] Alberto Molinari, Angelo Montanari, Aniello Murano, Giuseppe Perelli \& Adriano Peron (2016): Checking interval properties of computations. Acta Inf. 53(6-8), pp. 587-619, doi:10.1007/s00236-015-0250-1.

[14] Angelo Montanari \& Pietro Sala (2012): An Optimal Tableau System for the Logic of Temporal Neighborhood over the Reals. In Ben C. Moszkowski, Mark Reynolds \& Paolo Terenziani, editors: 19th International Symposium on Temporal Representation and Reasoning, TIME 2012, Leicester, United Kingdom, September 12-14, 2012, IEEE Computer Society, pp. 39-46, doi:10.1109/TIME.2012.18.

[15] Sylvain Schmitz (2016): Complexity Hierarchies Beyond Elementary. ACM Transactions on Computation Theory 8(1), pp. 3:1-3:36, doi:10.1145/2858784.

[16] Larry Joseph Stockmeyer (1974): The complexity of decision problems in automata theory and logic. Ph.D. thesis, Massachusetts Institute of Technology.

[17] Yde Venema (1991): A Modal Logic for Chopping Intervals. Journal of Logic and Computation 1(4), pp. 453-476, doi:10.1093/logcom/1.4.453. 